\title{
Some New Kulli-Basava Topological Indices
}

\section{R. Kulli}

Department of Mathematics, Gulbarga University, Gulbarga 585106, India; e-mail: vrkulli@gmail.com

\begin{abstract}
Recently, Kulli-Basava indices were introduced and studied their mathematical and chemical properties which have good response with mean isomer degeneracy. In this paper, we introduce the modified first and second Kulli-Basava indices, $F_{1}$-Kulli-Basava index, square Kulli-Basava index of a graph, and compute exact formulas for regular graphs, wheels, gear graphs and helm graphs.
\end{abstract}

\section{Introduction}

Throughout this paper $G$ is a finite, simple, connected graph with vertex set $V(G)$ and edge set $E(G)$. The degree $d_{G}(v)$ of a vertex $v$ is the number of vertices adjacent to $v$. Let $|V(G)|=n$ and $|E(G)|=m$. The degree of an edge $e=u v$ in $G$ is defined by $d_{G}(e)=d_{G}(u)+d_{G}(v)-2$. Let $S_{e}(v)$ denote the sum of degrees of all edges incident to a vertex $v$. We refer to [1] for undefined term and notation.

Recently, the first and second Kulli-Basava indices were introduced in [2], defined as

$$
K B_{1}(G)=\sum_{u v \in E(G)}\left[S_{e}(u)+S_{e}(v)\right], \quad K B_{2}(G)=\sum_{u v \in E(G)} S_{e}(u) S_{e}(v) .
$$

Received: June 8, 2019; Accepted: July 20, 2019

2010 Mathematics Subject Classification: 05C05, 05C07, 05 C76.

Keywords and phrases: modified first and second Kulli-Basava indices, $F_{1}$-Kulli-Basava and square KulliBasava indices, graphs.

Copyright (C) 2019 V. R. Kulli. This is an open access article distributed under the Creative Commons Attribution License, which permits unrestricted use, distribution, and reproduction in any medium, provided the original work is properly cited. 
We introduce the modified first and second Kulli-Basava indices, defined as

$$
{ }^{m} K B_{1}(G)=\sum_{u v \in E(G)} \frac{1}{S_{e}(u)+S_{e}(v)}, \quad{ }^{m} K B_{2}(G)=\sum_{u v \in E(G)} \frac{1}{S_{e}(u) S_{e}(v)} .
$$

In [3], Furtula and Gutman studied the $F$-index, defined as

$$
F(G)=\sum_{u v \in E(G)}\left[S_{e}(u)^{2}+S_{e}(v)^{2}\right]
$$

Recently, the square ve-degree index was introduced by Kulli [4], defined as

$$
Q_{v e}(G)=\sum_{u v \in E(G)}\left[d_{v e}(u)-d_{v e}(v)\right]^{2}
$$

We now propose the $F_{1}$-Kulli-Basava and square Kulli-Basava indices, defined as

$$
\begin{aligned}
F_{1} K B(G) & =\sum_{u v \in E(G)}\left[S_{e}(u)^{2}+S_{e}(v)^{2}\right], \\
Q K B(G) & =\sum_{u v \in E(G)}\left[S_{e}(u)-S_{e}(v)\right]^{2} .
\end{aligned}
$$

Recently, some $F$-indices were studied, for example, in $[5,6,7,8,9,10,11]$ and also some square indices were studied, for example, in [12, 13, 14, 15, 16].

We introduce the $F_{1}$-Kulli-Basava polynomial and square Kulli-Basava polynomial of a graph, defined as

$$
\begin{aligned}
& F_{1} K B(G, x)=\sum_{u v \in E(G)} x^{S_{e}(u)^{2}+S_{e}(v)^{2}}, \\
& Q K B(G, x)=\sum_{u v \in E(G)} x^{\left[S_{e}(u)-S_{e}(v)\right]^{2}} .
\end{aligned}
$$

In this paper, we establish explicit formulas for the modified first and second KulliBasava indices, $F_{1}$-Kulli-Basava and square Kulli-Basava indices of some graphs. Also the $F_{1}$-Kulli-Basava and square Kulli-Basava polynomials of some graphs are obtained. 


\section{Regular Graphs}

Theorem 1. Let $G$ be an r-regular graph with $n$ vertices and $m$ edges. Then

(i) ${ }^{m} K B_{1}(G)=\frac{m}{4 r(r-1)}$.

(ii) ${ }^{m} K B_{2}(G)=\frac{m}{4 r^{2}(r-1)^{2}}$.

(iii) $F_{1} K B(G)=8 m r^{2}(r-1)^{2}$.

(iv) $Q K B(G)=0$.

Proof. Let $G$ be an $r$-regular graph with $n$ vertices. Then $S_{e}(u)=2 r(r-1)$ for any vertex $u$ in $G$.

Thus

(i) ${ }^{m} K B_{1}(G)=\sum_{u v \in E(G)} \frac{1}{S_{e}(u)+S_{e}(v)}=\frac{m}{2 r(r-1)+2 r(r-1)}=\frac{m}{4 r(r-1)}$.

(ii) ${ }^{m} K B_{2}(G)=\sum_{u v \in E(G)} \frac{1}{S_{e}(u) S_{e}(v)}=\frac{m}{2 r(r-1) 2 r(r-1)}=\frac{m}{4 r^{2}(r-1)^{2}}$.

(iii) $F_{1} K B(G)=\sum_{u v \in E(G)}\left[S_{e}(u)^{2}+S_{e}(v)^{2}\right]=m\left[(2 r(r-1))^{2}+(2 r(r-1))^{2}\right]$

$$
=8 m r^{2}(r-1)^{2} \text {. }
$$

(iv) $Q K B(G)=\sum_{u v \in E(G)}\left(S_{e}(u)-S_{e}(v)\right)^{2}=0$.

Corollary 1.1. If $C_{n}$ is a cycle with $n$ vertices, then
(i) ${ }^{m} K B_{1}\left(C_{n}\right)=\frac{n}{8}$.
(ii) ${ }^{m} K B_{2}\left(C_{n}\right)=\frac{n}{16}$.
(iii) $F_{1} K B\left(C_{n}\right)=32 n$.
(iv) $Q K B\left(C_{n}\right)=0$.

Corollary 1.2. If $K_{n}$ is a complete graph with $n$ vertices, then
(i) ${ }^{m} K B_{1}\left(K_{n}\right)=\frac{n}{8(n-2)}$.
(ii) ${ }^{m} K B_{2}\left(K_{n}\right)=\frac{n}{8(n-1)(n-2)^{2}}$.
(iii) $F_{1} K B\left(K_{n}\right)=4 n(n-1)^{3}(n-2)^{2}$.
(iv) $Q K B\left(K_{n}\right)=0$. 
Theorem 2. If $G$ is an r-regular graph with $n$ vertices and $m$ edges, then

(i) $F_{1} K B(G, x)=m x^{8 r^{2}(r-1)^{2}}$.

(ii) $Q K B(G, x)=m x^{0}$.

Proof. Let $G$ be an $r$-regular graph with $n$ vertices and $m$ edges. Then $S_{e}(u)=2 r(r-1)$ for $u \in V(G)$. Thus

(i) $F_{1} K B(G, x)=\sum_{u v \in E(G)} x^{S_{e}(u)^{2}+S_{e}(v)^{2}}=m x^{(2 r(r-1))^{2}+(2 r(r-1))^{2}}=m x^{8 r^{2}(r-1)^{2}}$.

(ii) $Q K B(G, x)=\sum_{u v \in E(G)} x^{\left[S_{e}(u)-S_{e}(v)\right]^{2}}=m x^{0}$.

Corollary 2.1. If $C_{n}$ is a cycle with $n$ vertices, then
(i) $F_{1} K B\left(C_{n}, x\right)=n x^{32}$.
(ii) $Q K B\left(C_{n}, x\right)=n x^{0}$.

Corollary 2.2. If $K_{n}$ is a complete graph with $n$ vertices, then

(i) $F_{1} K B\left(K_{n}, x\right)=\frac{n(n-1)}{2} x^{8(n-1)^{2}(n-2)^{2}}$.

(ii) $Q K B\left(K_{n}, x\right)=\frac{n(n-1)}{2} x^{0}$.

\section{Wheel Graphs}

A wheel $W_{n}$ is the join of $K_{1}$ and $C_{n}$. Clearly $W_{n}$ has $n+1$ vertices and $2 n$ edges. A wheel $W_{n}$ is presented in Figure 1. The vertices of $C_{n}$ are called rim vertices and the vertex of $K_{1}$ is called apex.

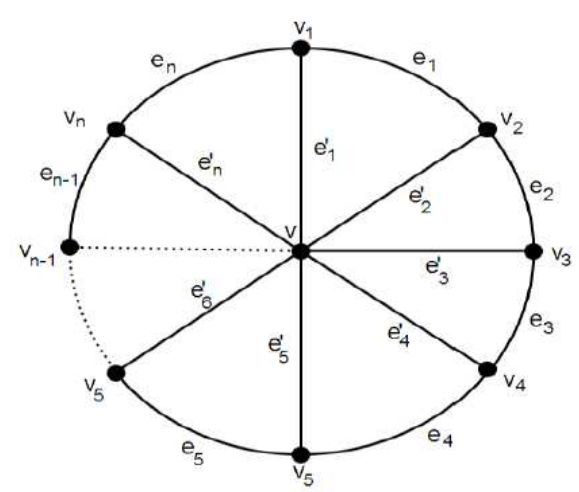

Figure 1. Wheel $W_{n}$. 
Lemma 1. Let $W_{n}$ be a wheel with $n+1$ vertices and $2 n$ edges, $n \geq 3$. Then

$$
\begin{array}{ll}
E_{1}=\left\{u v \in E\left(W_{n}\right) \mid S_{e}(u)=n+9,\left(S_{e}(v)=n(n+1)\right)\right\}, & \left|E_{1}\right|=n \\
E_{2}=\left\{u v \in E\left(W_{n}\right) \mid S_{e}(u)=n+9,\left(S_{e}(v)=n+9\right)\right\}, & \left|E_{2}\right|=n .
\end{array}
$$

Theorem 3. Let $W_{n}$ be a wheel with $n+1$ vertices and $2 n$ edges, $n \geq 3$. Then

(i) ${ }^{m} K B_{1}\left(W_{n}\right)=\frac{n}{n^{2}+2 n+9}+\frac{n}{2 n+18}$.

(ii) ${ }^{m} K B_{2}\left(W_{n}\right)=\frac{1}{(n+9)(n+1)}+\frac{n}{(n+9)^{2}}$.

(iii) $F_{1} K B\left(W_{n}\right)=\left(n^{3}+5 n^{2}+55 n+243\right)$.

(iv) $Q K B\left(W_{n}\right)=n\left(n^{2}-9\right)^{2}$.

Proof. By using definitions and Lemma 1, we derive

(i) ${ }^{m} K B_{1}\left(W_{n}\right)=\sum_{u v \in E\left(W_{n}\right)} \frac{1}{S_{e}(u)+S_{e}(v)}$

$$
\begin{aligned}
& =\left|E_{1}\right|\left(\frac{1}{n+9+n(n+1)}\right)+\left|E_{2}\right|\left(\frac{1}{n+9+n+9}\right) \\
& =\frac{n}{n^{2}+2 n+9}+\frac{n}{2 n+18} .
\end{aligned}
$$

(ii) ${ }^{m} K B_{2}\left(W_{n}\right)=\sum_{u v \in E\left(W_{n}\right)} \frac{1}{S_{e}(u) S_{e}(v)}$

$$
\begin{aligned}
& =\left|E_{1}\right|\left(\frac{1}{(n+9) \times n(n+1)}\right)+\left|E_{2}\right|\left(\frac{1}{(n+9)(n+9)}\right) \\
& =\frac{1}{(n+9)(n+1)}+\frac{n}{(n+9)^{2}} .
\end{aligned}
$$

(iii) $F_{1} K B\left(W_{n}\right)=\sum_{u v \in E\left(W_{n}\right)}\left[S_{e}(u)^{2}+S_{e}(v)^{2}\right]$ 


$$
\begin{aligned}
& =\left|E_{1}\right|\left[(n+9)^{2}+(n(n+1))^{2}\right]+\left|E_{2}\right|\left[(n+9)^{2}+(n+9)^{2}\right] \\
& =n\left(n^{3}+5 n^{2}+55 n+243\right) . \\
\text { (iv) } Q K B\left(W_{n}\right) & =\sum_{u v \in E\left(W_{n}\right)}\left[S_{e}(u)-S_{e}(v)\right]^{2} \\
& =\left|E_{1}\right|[(n+9)-(n(n+1))]^{2}+\left|E_{2}\right|[(n+9)-(n+9)]^{2} \\
& =n\left(n^{2}-9\right)^{2} .
\end{aligned}
$$

Theorem 4. Let $W_{n}$ be a wheel with $n+1$ vertices and $2 n$ edges, $n \geq 3$. Then

(i) $F_{1} K B\left(W_{n}, x\right)=n x^{n^{3}+3 n^{2}+19 n+81}+n x^{2(n+9)^{2}}$.

(ii) $Q K B\left(W_{n}, x\right)=n x^{\left(n^{2}-9\right)^{2}}+n x^{0}$.

Proof. By using definitions and Lemma 1, we deduce

$$
\text { (i) } \begin{aligned}
F_{1} K B\left(W_{n}, x\right) & =\sum_{u v \in E\left(W_{n}\right)} x^{\left[S_{e}(u)^{2}+S_{e}(v)^{2}\right]} \\
& =\left|E_{1}\right| x^{(n+9)^{2}+n^{2}(n+1)^{2}}+\left|E_{2}\right| x^{(n+9)^{2}+(n+9)^{2}} \\
& =n x^{n^{3}+3 n^{2}+19 n+81}+n x^{2(n+9)^{2}}
\end{aligned}
$$

(ii) $Q K B\left(W_{n}, x\right)=\sum_{u v \in E\left(W_{n}\right)} x^{\left[S_{e}(u)-S_{e}(v)\right]^{2}}$

$$
\begin{aligned}
& =\left|E_{1}\right| x^{[n+9-n(n+1)]^{2}}+\left|E_{2}\right| x^{[(n+9)-(n+9)]^{2}} \\
& =n x^{\left(n^{2}-9\right)^{2}}+n x^{0} .
\end{aligned}
$$

\section{Gear Graphs}

A graph is a gear graph obtained from $W_{n}$ by adding a vertex between each pair of adjacent rim vertices and it is denoted by $G_{n}$. Clearly $G_{n}$ has $2 n+1$ vertices and $3 n$ edges. A graph $G_{n}$ is depicted in Figure 2. 


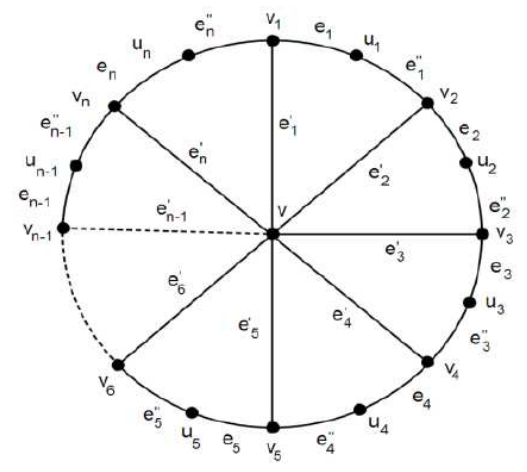

Figure 2. Gear graph $G_{n}$.

Lemma 2. Let $G_{n}$ be a gear graph with $3 n$ edges. Then $G_{n}$ has two types of edges as follows:

$$
\begin{array}{ll}
E_{1}=\left\{u v \in E\left(G_{n}\right) \mid S_{e}(u)=n(n+1), S_{e}(v)=n+7\right\}, & \left|E_{1}\right|=n . \\
E_{2}=\left\{u v \in E\left(G_{n}\right) \mid S_{e}(u)=n+7, S_{e}(v)=6\right\}, & \left|E_{2}\right|=2 n .
\end{array}
$$

Theorem 5. If $G_{n}$ is a gear graph with $2 n+1$ vertices and $3 n$ edges, then

(i) ${ }^{m} K B_{1}\left(G_{n}\right)=\frac{n}{n^{2}+2 n+7}+\frac{2 n}{n+13}$.

(ii) ${ }^{m} K B_{2}\left(G_{n}\right)=\frac{1}{(n+1)(n+7)}+\frac{n}{3(n+7)}$.

(iii) $F_{1} K B\left(G_{n}\right)=n\left(n^{4}+2 n^{3}+4 n^{2}+42 n+219\right)$.

(iv) $Q K B\left(G_{n}\right)=n\left(n^{4}-12 n^{2}+4 n+51\right)$.

Proof. By using definitions and Lemma 2, we deduce

$$
\text { (i) } \begin{aligned}
{ }^{m} K B_{1}\left(G_{n}\right) & =\sum_{u v \in E\left(G_{n}\right)} \frac{1}{S_{e}(u)+S_{e}(v)} \\
& =\left|E_{1}\right|\left(\frac{1}{n(n+1)+(n+7)}\right)+\left|E_{2}\right|\left(\frac{1}{n+7+6}\right) \\
& =\frac{n}{n^{2}+2 n+7}+\frac{2 n}{n+13} .
\end{aligned}
$$




$$
\text { (ii) } \begin{aligned}
{ }^{m} K B_{2}\left(G_{n}\right) & =\sum_{u v \in E\left(G_{n}\right)} \frac{1}{S_{e}(u) S_{e}(v)} \\
& =\left|E_{1}\right|\left(\frac{1}{n(n+1)(n+7)}\right)+\left|E_{2}\right|\left(\frac{1}{(n+7) 6}\right) \\
& =\frac{1}{(n+1)(n+7)}+\frac{n}{3(n+7)} .
\end{aligned}
$$

$$
\text { (iii) } \begin{aligned}
F_{1} K B\left(G_{n}\right) & =\sum_{u v \in E\left(G_{n}\right)}\left[S_{e}(u)^{2}+S_{e}(v)^{2}\right] \\
& =\left|E_{1}\right|\left[\left(n^{2}+n\right)^{2}+(n+7)^{2}\right]+\left|E_{2}\right|\left[(n+7)^{2}+6^{2}\right] \\
& =n\left(n^{4}+2 n^{3}+4 n^{2}+42 n+219\right) .
\end{aligned}
$$

$$
\text { (iv) } \begin{aligned}
Q K B\left(G_{n}\right) & =\sum_{u v \in E\left(G_{n}\right)}\left[S_{e}(u)-S_{e}(v)\right]^{2} \\
& =\left|E_{1}\right|\left(n^{2}+n-n-7\right)^{2}+\left|E_{2}\right|(n+7-6)^{2} \\
& =n\left(n^{4}-12 n^{2}+4 n+51\right) .
\end{aligned}
$$

Theorem 6. Let $G_{n}$ be a gear graph with $2 n+1$ vertices and $3 n$ edges, $n \geq 3$. Then

(i) $F_{1} K B\left(G_{n}, x\right)=n x^{n^{3}+3 n^{2}+15 n+49}+2 n x^{n^{2}+14 n+85}$.

(ii) $Q K B\left(G_{n}, x\right)=n x^{\left(n^{2}-7\right)^{2}}+2 n x^{(n+1)^{2}}$.

Proof. By using definitions and Lemma 2, we obtain

$$
\text { (i) } \begin{aligned}
F_{1} K B\left(G_{n}, x\right) & =\sum_{u v \in E\left(G_{n}\right)} x^{\left[S_{e}(u)^{2}+S_{e}(v)^{2}\right]} \\
& =\left|E_{1}\right| x^{\left(n^{2}+n\right)^{2}+(n+7)^{2}}+\left|E_{2}\right| x^{(n+7)^{2}+6^{2}} \\
& =n x^{n^{3}+3 n^{2}+15 n+49}+2 n x^{n^{2}+14 n+85}
\end{aligned}
$$




$$
\text { (ii) } \begin{aligned}
Q K B\left(G_{n}, x\right) & =\sum_{u v \in E\left(G_{n}\right)} x^{\left[S_{e}(u)-S_{e}(v)\right]^{2}} \\
& =\left|E_{1}\right| x^{\left(n^{2}+n-n-7\right)^{2}}+\left|E_{2}\right| x^{(n+7-6)^{2}} \\
& =n x^{\left(n^{2}-7\right)^{2}}+2 n x^{(n+1)^{2}} .
\end{aligned}
$$

\section{Helm Graphs}

A helm graph $H_{n}$ is a graph obtained from $W_{n}$ by attaching an end edge to each rim vertex. Clearly $H_{n}$ has $2 n+1$ vertices and $3 n$ edges. A graph $H_{n}$ is shown in Figure 3.

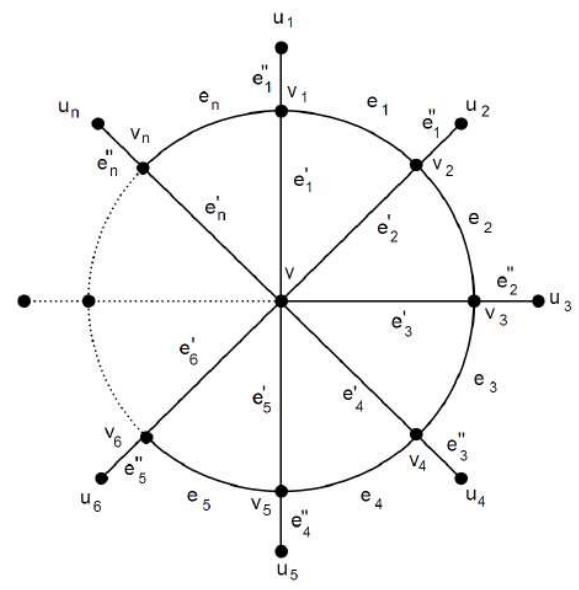

Figure 3. Helm graph $H_{n}$.

Lemma 3. Let $H_{n}$ be a helm graph with $3 n$ edges. Then $H_{n}$ has three types of edges as given below:

$$
\begin{array}{ll}
E_{1}=\left\{u v \in E\left(H_{n}\right) \mid S_{e}(u)=n(n+2), S_{e}(v)=n+17\right\}, & \left|E_{1}\right|=n . \\
E_{2}=\left\{u v \in E\left(H_{n}\right) \mid S_{e}(u)=S_{e}(v)=n+17\right\}, & \left|E_{2}\right|=n . \\
E_{3}=\left\{u v \in E\left(H_{n}\right) \mid S_{e}(u)=n+17, S_{e}(v)=3\right\}, & \left|E_{3}\right|=n .
\end{array}
$$

Theorem 7. Let $H_{n}$ be a helm graph with $2 n+1$ vertices and $3 n$ edges. Then

(i) ${ }^{m} K B_{1}\left(H_{n}\right)=\frac{n}{n^{2}+3 n+17}+\frac{n}{2 n+34}+\frac{n}{n+20}$. 
(ii) ${ }^{m} K B_{2}\left(H_{n}\right)=\frac{1}{(n+2)(n+17)}+\frac{n}{(n+17)^{2}}+\frac{n}{3(n+17)}$.

(iii) $F K B\left(H_{n}\right)=n\left[n^{2}(n+2)^{2}+(n+17)^{2}\right]+2 n(n+17)^{2}+n\left[(n+17)^{2}+9\right]$.

(iv) $Q K B\left(H_{n}\right)=n\left(n^{2}+n-17\right)^{2}+n(n+14)^{2}$.

Proof. By using definitions and Lemma 3, we deduce

$$
\text { (i) } \begin{aligned}
{ }^{m} K B_{1}\left(H_{n}\right)= & \sum_{u v \in E\left(H_{n}\right)} \frac{1}{S_{e}(u)+S_{e}(v)} \\
= & \left|E_{1}\right|\left(\frac{1}{n(n+2)+n+17}\right)+\left|E_{2}\right|\left(\frac{1}{n+17+n+17}\right) \\
& +\left|E_{3}\right|\left(\frac{1}{n+17+3}\right) \\
= & \frac{n}{n^{2}+3 n+17}+\frac{n}{2 n+34}+\frac{n}{n+20} .
\end{aligned}
$$

(ii) ${ }^{m} K B_{2}\left(H_{n}\right)=\sum_{u v \in E\left(H_{n}\right)} \frac{1}{S_{e}(u) S_{e}(v)}$

$$
\begin{aligned}
= & \left|E_{1}\right|\left(\frac{1}{n(n+2)(n+17)}\right)+\left|E_{2}\right|\left(\frac{1}{(n+17)(n+17)}\right) \\
& +\left|E_{3}\right|\left(\frac{1}{(n+17) 3}\right) \\
= & \frac{1}{(n+2)(n+17)}+\frac{n}{(n+17)^{2}}+\frac{n}{3(n+17)} .
\end{aligned}
$$

$$
\text { (iii) } \begin{aligned}
F_{1} K B\left(H_{n}\right)= & \sum_{u v \in E\left(H_{n}\right)}\left[S_{e}(u)^{2}+S_{e}(v)^{2}\right] \\
= & \left|E_{1}\right|\left[n^{2}(n+2)^{2}+(n+17)^{2}\right]+\left|E_{2}\right|\left[(n+17)^{2}+(n+17)^{2}\right] \\
& +\left|E_{3}\right|\left[(n+17)^{2}+3^{2}\right] \\
= & n\left[n^{2}(n+2)^{2}+(n+17)^{2}\right]+2 n(n+17)^{2}+n\left[(n+17)^{2}+9\right]
\end{aligned}
$$




$$
\text { (iv) } \begin{aligned}
\operatorname{QKB}\left(H_{n}\right)= & \sum_{u v \in E\left(H_{n}\right)}\left[S_{e}(u)-S_{e}(v)\right]^{2} \\
= & \left|E_{1}\right|\left(n^{2}+2 n-n-17\right)^{2}+\left|E_{2}\right|(n+17-n-17)^{2} \\
& +\left|E_{3}\right|(n+17-3)^{2} \\
= & n\left(n^{2}+n-17\right)+n(n+14)^{2} .
\end{aligned}
$$

Theorem 8. Let $H_{n}$ be a helm graph with $2 n+1$ vertices and $3 n$ edges. Then

(i) $F_{1} K B\left(H_{n}, x\right)=n x^{n^{2}(n+2)^{2}+(n+17)^{2}}+n x^{2(n+17)^{2}}+n x^{(n+17)^{2}+9}$.

(ii) $Q K B\left(H_{n}, x\right)=n x^{\left(n^{2}+n-17\right)^{2}}+n x^{0}+n x^{(n+14)^{2}}$.

Proof. By using definitions and Lemma 3, we obtain

$$
\text { (i) } \begin{aligned}
F_{1} K B\left(H_{n}, x\right)= & \sum_{u v \in E\left(H_{n}\right)} x^{\left[S_{e}(u)^{2}+S_{e}(v)^{2}\right]} \\
= & \left|E_{1}\right| x^{n^{2}(n+2)^{2}+(n+17)^{2}}+\left|E_{2}\right| x^{(n+17)^{2}+(n+17)^{2}} \\
& +\left|E_{3}\right| x^{(n+17)^{2}+3^{2}} \\
= & n x^{n^{2}(n+2)^{2}+(n+17)^{2}}+n x^{2(n+17)^{2}}+n x^{(n+17)^{2}+9} .
\end{aligned}
$$

(ii) $\operatorname{QKB}\left(H_{n}, x\right)=\sum_{u v \in E\left(H_{n}\right)} x^{\left[S_{e}(u)-S_{e}(v)\right]^{2}}$

$$
\begin{aligned}
& =\left|E_{1}\right| x^{\left(n^{2}+2 n-n-17\right)^{2}}+\left|E_{2}\right| x^{(n+17-n-17)^{2}}+\left|E_{3}\right| x^{(n+17-3)^{2}} \\
& =n x^{\left(n^{2}+n-17\right)^{2}}+n x^{0}+n x^{(n+14)^{2}} .
\end{aligned}
$$

\section{References}

[1] V. R. Kulli, College Graph Theory, Vishwa International Publications, Gulbarga, India, 2012.

[2] B. Basavanagoud and P. Jakkannavar, Kulli-Basava indices of graphs, Inter. J. Appl. Engg. Research 14(1) (2018), 325-342. 
[3] B. Furtula and I. Gutman, A forgotten topological index, J. Math. Chem. 53 (2015), 11841190. https://doi.org/10.1007/s10910-015-0480-z

[4] V. R. Kulli, On the square ve-degree index and its polynomial of certain network, Journal of Global Research in Mathematical Archives 5(10) (2018), 1-4.

[5] N. De, F-index of bridge and chain graphs, Malay. J. Fund. Appl. Sci. 12 (2016), 109113. https://doi.org/10.11113/mjfas.v12n3.471

[6] S. Ghobadi and M. Ghorbaninejad, The forgotten topological index for four operations on some special graphs, Bull. Math. Sci. Appl. 16 (2016), 89-95. https://doi.org/10.18052/www.scipress.com/BMSA.16.89

[7] V. R. Kulli, $F$-Revan index and $F$-Revan polynomial of some families of benzenoid systems, Journal of Global Research in Mathematical Archives 5(11) (2018), 1-6.

[8] V. R. Kulli, Computing square Revan index and its polynomial of certain benzenoid systems, International Journal of Mathematical Archive 9(12) (2018), 41-49.

[9] V. R. Kulli, Computing $F$-reverse index and $F$-reverse polynomial of certain networks, International Journal of Mathematical Archive 9(8) (2018), 27-33.

[10] V. R. Kulli, Computing the $F$-ve-degree index and its polynomial of dominating oxide and regular triangulate oxide networks, International Journal of Fuzzy Mathematical Archive 16(1) (2018), 1-6.

[11] V. R. Kulli, Neighborhood Dakshayani indices, submitted.

[12] V. R. Kulii, Computing square Revan index and its polynomial of certain benzenoid systems, International Journal of Mathematics and its Applications 6(4) (2018), 213-219.

[13] V. R. Kulli, Square reverse index and its polynomial of certain networks, International Journal of Mathematical Archive 9(10) (2018), 27-33.

[14] V. R. Kulli, Minus leap and square leap indices and their polynomials of some special graphs, International Research Journal of Pure Algebra 8(11) (2018), 54-60.

[15] V. R. Kulli, On hyper $K V$ and square $K V$ indices and their polynomials of certain families of dendrimers, Journal of Computer and Mathematical Sciences 10(2) (2019), 279-286. https://doi.org/10.29055/jcms/1007

[16] V. R. Kulli, Minus $F$ and square $F$-indices and their polynomials of certain dendrimers, Earthline J. Math. Sci. 1(2) (2019), 171-185. https://doi.org/10.34198/ejms.1219.171185 